\section{LA INFLUENCIA DE LA FAMILIA EN EL OCIO FÍSICO-DEPORTIVO JUVENIL: NUEVAS PERSPECTIVAS PARA LA REFLEXIÓN Y LA ACCIÓN}

\author{
María Ángeles Valdemoros-San-Emeterio \\ Universidad de La Rioja \\ maria-de-los-angeles.valdemoros@unirioja.es \\ Ana Ponce-de-León-Elizondo \\ Universidad de La Rioja \\ ana.ponce@unirioja.es \\ Eva Sanz-Arazuri \\ Universidad de La Rioja \\ eva.sanz@unirioja.es \\ José Antonio Caride-Gómez \\ Universidad de Santiago de Compostela \\ joseantonio.caride@usc.es
}

Cómo citar este artículo/Citation: Valdemoros-San-Emeterio, M. A.; Ponce-de-León-Elizondo, A.; Sanz-Arazuri, E. y CarideGómez, J. A. (2014). "La influencia de la familia en el ocio físico-deportivo juvenil: nuevas perspectivas para la reflexión y la acción". Arbor, 190 (770): a192. doi: http://dx.doi. org/10.3989/arbor.2014.770n6013

Recibido: 4 diciembre 2013. Aceptado: 3 septiembre 2014.

RESUMEN: La familia influye decisivamente en la construcción del ocio juvenil, como ámbito de convivencia en el que las personas concretan los modos de disfrutar de sus tiempos libres y como agente principal en las prácticas de ocio, atendiendo a distintos factores o circunstancias; entre otros, el funcionamiento interno familiar aparece como uno de los condicionantes más relevantes. La revisión de la literatura científica manifiesta hallazgos contradictorios que debilitan las teorías que señalan al estilo educativo familiar, nivel educativo y/o económico, comportamiento modelo de los padres o estructura familiar como factores influyentes en el ocio físico-deportivo de los hijos adolescentes, mientras fortalecen aquellas que apuntan como variables condicionantes la cohesión, comunicación y flexibilidad, que conforman el funcionamiento familiar. Estas conclusiones avalan la necesidad de profundizar en estos factores, así como en el diseño de programas de acción-intervención-educación familiar que potencien la autonomía y responsabilidad de los jóvenes sobre su propio ocio.

PALABRAS CLAVE: Educación del ocio; jóvenes; familia; actividad física.
THE INFLUENCE OF THE FAMILY IN THE PHYSICAL SPORTIVE JUVENILE LEISURE: NEW PERSPECTIVES FOR THE REFLECTION AND THE ACTION
Copyright: (C) 2014 CSIC. Este es un artículo de acceso abierto distribuido bajo los términos de la licencia Creative Commons Attribution-Non Commercial (by-nc) Spain 3.0.
ABSTRACT: The family has a decisive influence on the construction of youth leisure, as the domain of coexistence in which people spend their free time and as a main agent in leisure activities. The manner of this influence depends on various factors or circumstances, among which how the family functions internally emerges as one of the most important. Scientific research has produced contradictory findings, undermining theories that the family's style of upbringings, educational and/or economic level, parental behaviour and family structure are influential factors in teenagers' physical and sports leisure, while bolstering the idea of the importance of family cohesion, communication and flexibility in the way the family functions. Further study of these factors is therefore warranted, and family action-intervention-education programmes need to be designed to boost young people's autonomy and responsibility with regard to their leisure

KEYWORDS: Leisure education; youth; family; physical activity. 


\section{INTRODUCCIÓN: ENTRE LA FAMILIA Y EL OCIO}

La sociedad de la información y la comunicación nos ha situado ante nuevas necesidades que obligan a realizar reajustes en la organización, planificación y gestión de los tiempos sociales, incluido el ocio.

Por un lado, las familias deben adaptar y/o conciliar sus ritmos a circunstancias laborales, educativas y sociales que afectan, directa o indirectamente, al conjunto de sus miembros, sin que -con demasiada frecuencia- sea factible acomodar la elección de la distribución deseada a las expectativas o realidades que emergen de la vida cotidiana.

Por otro lado, fuera del ámbito familiar, con el fin de evitar que el tiempo libre derive en un ocio nocivo para los jóvenes, se constata el creciente afán por conocer más y mejor sus problemáticas específicas, así como proponer alternativas que las afronten, cada vez más condicionadas por la cultura del consumo y las dificultades que existen para articular y/o armonizar los horarios familiares con los escolares, laborales y sociales.

Estas realidades justifican el interés social y la relevancia científica de llevar a cabo estudios como el presente, que indaga en aquellas variables familiares susceptibles de contribuir a la construcción del ocio juvenil como fuente de desarrollo humano $y$, más en concreto, de la actividad física de ocio como ámbito privilegiado para el crecimiento personal y el bienestar social.

El ocio inscribe sus realidades en espacios y tiempos especialmente significativos para el desarrollo humano, de cada persona y de la sociedad en su conjunto (McDavid, Cox y Amorose, 2011; Van der Horst, Oenema, Te Velde y Brug, 2010).

De partida, todo indica que en el logro de un clima familiar propicio para la construcción de un ocio físico-deportivo, que favorezca el desarrollo armónico de los jóvenes, interviene decisivamente en la organización y distribución de los tiempos familiares: en la asunción de las responsabilidades comunes y diferenciadas de cada uno de sus miembros, la atención a las necesidades cotidianas, la transición del trabajo al descanso, las obligaciones del cuidado personal y colectivo, etc. En ellos se constata que los ámbitos laboral, doméstico y escolar son los que más dificultan la conciliación entre quienes participan de sus dinámicas -padres e hijos, fundamentalmente- lo que obliga a revisar el protagonismo que les corresponde en las actuaciones que se llevan a cabo, cada vez más afectados por los complejos y acelerados cambios que se están produciendo en la sociedad de la información y la comunicación (Caballo, Gradaílle y Merelas, 2012; Fraguela, Lorenzo y Varela, 2011; Torres, 2007). La falta de tiempo, argumenta Schnabel (2011, p.19), "es el mayor mal de la sociedad moderna. Ya seamos autónomos o empleados, directivos, políticos o científicos, a todos nos une la sensación de estar permanentemente bajo presión".

Aunque se trata de un problema heredado, las conexiones entre el mundo laboral y familiar inducen a pensar mucho más en una relación de contradicción que de conciliación (Tobío, 2002), exigiendo que las familias adapten sus ritmos temporales a circunstancias laborales, educativas y sociales cada vez más adversos para la convivencia entre sus miembros. Las complicaciones inherentes a la crisis socioeconómica, supeditando la dinámica familiar a decisiones externas o limitando los recursos disponibles o accesibles en su entorno, han incrementado significativamente las dificultades para conciliar los deseos personales con la vida en común y, de un modo específico, la jornada laboral con los tiempos sociales que habilitan las escuelas, el transporte, el comercio, las prácticas deportivas y culturales, etc.

Fuera del ámbito familiar, lo que ha dado en Ilamarse "ocio nocivo" constituye una de las principales preocupaciones de las sociedades del desarrollo (marcado por el consumo), de modo que la búsqueda de soluciones a algunos de los problemas que en ellas se plantean, como son el polémico "botellón", la cultura consumista, el individualismo o el aburrimiento, pasan por el ejercicio de un otium cum dignitate -ocio con dignidad- acomodado a los nuevos tiempos (Cuenca, 2002). Un ocio que obliga no solo a conocer más y mejor las realidades de los jóvenes y adolescentes sino, también, a proponer alternativas que afronten sus problemáticas específicas, en las que las familias son uno de los principales agentes educativos, sobre todo por las influencias que los valores, actitudes, hábitos o comportamientos generan en las vivencias individuales y colectivas de sus integrantes, ya desde la primera infancia.

La literatura existente, que analiza los vínculos entre diferentes tipologías familiares (socioeconómica, cultural, educativa, laboral, convivencial, etc.) y el ocio físico-deportivo de sus jóvenes, ofrece resultados discrepantes (Cantallops et al., 2012; Carlson et al., 2010; Fuemmeler, Anderson y Mâsse, 2011; Gorely et al., 2009; Kahn et al., 2008; Lin, Lin y Wu, 2009; Pérez Milena et al., 2007; Piéron y Ruiz Juan, 
2013; Piko y Keresztes, 2008; Rangul et al., 2011; Richards et al., 2009; Ruiz-Juan, Pierón y Baena-Extremera, 2012; Sanz, Ponce de León y Valdemoros, 2012; Sharp et al., 2006; Thérond, Duyme y Capron, 2002; Toftegaard-Stockel et al., 2011; Tuero, Zapico y González, 2012; Van der Horst et al., 2010; Van der Horst et al., 2007).

Su análisis sugiere plantear, como hipótesis de partida, que los factores relacionados con el funcionamiento interno familiar son los verdaderos condicionantes en la construcción del ocio físico-deportivo juvenil, quedando relegados otros de alcance educativo, socioeconómico, cultural, estructural o laboral.

En este sentido, formulamos como un objetivo explícito de nuestra contribución revisar la literatura científica -nacional e internacional- que toma como referencia las variables familiares influyentes en la práctica físico-deportiva juvenil en los espaciostiempos de ocio, sin obviar el importante papel que en ellos tiene o debe tener la educación.

Para llevar a cabo dicha revisión se realizó una búsqueda sistemática en la base de datos electrónica Web of Knowledge, seleccionando como palabras clave las más empleadas científicamente para referirse a cada una de las tres fuentes de nuestro estudio: Funcionamiento familiar "family functioning", Ocio "leisure" y Actividad Física "physical activity", incidicendo en aquellos trabajos pertenecientes a la última década.

Con este propósito el texto se articula en cuatro apartados, comenzando por una breve reflexión acerca del ocio en la sociedad actual, que da paso a la consideración del ocio físico-deportivo como un ámbito privilegiado para el crecimiento-desarrollo personal, a la valoración de la institución familiar en la sociedad de la información y la comunicación y, por último, al análisis de las variables familiares susceptibles de influir en el ocio físico-deportivo juvenil (contexto educativo, pautas educativas y/o económicas, estilos de vida de los padres, estructura y funcionamiento familiar).

Las conclusiones derivadas de este análisis inscriben sus aportes en la apertura y/o profundización del quehacer investigador en la identificación y valoración de los factores familiares que influyen o podrán hacerlo en una doble perspectiva: de un lado, la que ponga en valor la construcción del ocio como una vivencia significativa para el desarrollo humano, a nivel individual y colectivo; de otro, la que promueva el diseño de programas de educación e interven- ción familiar que favorezcan el crecimiento personal de los jóvenes, al tiempo que contribuye a mejorar sus mecanismos de socialización y participación en la vida social, de forma cotidiana y con visión de futuro.

\section{LAS REALIDADES DEL OCIO Y SU EDUCACIÓN EN UNA SOCIEDAD DE REDES}

En la sociedad que inicia el siglo XXI, el tiempo de ocio ha pasado de ser una atractiva posibilidad a constituir un derecho en sí mismo, siendo estimado en mayor medida por el colectivo juvenil (Aristegui y Silvestre, 2012). A su vez, el ocio se enraiza en el ámbito axiológico, siendo algunos de sus valores distintivos la libertad, la satisfacción y la voluntariedad (Cuenca y Goytia, 2012). De ahí su trascendencia para cualquier desarrollo humano que se pretenda, integral e integrador, de todas las personas y del todo en cada persona: individual y colectivo, cognitivo y emocional (Caride, 1998; Cuenca, 1997, 2009; Quintana, 1991).

Recientes estudios sobre el ocio (Roberts, 2012) centran su atención en los cambios experimentados por los jóvenes europeos en esta dimensión sociobiográfica, poniendo de relieve como desde los años cincuenta del pasado siglo es una de las variables más influyentes en sus trayectorias vitales, afectando a la prolongación de la condición juvenil, a los estilos de vida o al consumo de determinados bienes o servicios, que la crisis socio-económica está modulando o reconduciendo, sobre todo en los colectivos de jóvenes que la padecen más directamente, ya sea a nivel personal o familiar.

En este escenario, Cuenca y Goytia (2012) revelan que la sociedad actual concede más oportunidades para la realización de experiencias de ocio, si bien cada individuo las usa de forma diferente, dependiendo de sus actitudes, intereses, competencias y capacidad de disfrute; cuestiones, según los autores, más relacionadas con su formación que con la oferta, por lo que el ocio experiencial de calidad requiere capacitación y, en consecuencia, un serio compromiso pedagógico (Caride, 2012).

A pesar de existir numerosas actividades de ocio interesantes y significativas, que configuran un marco idóneo para favorecer un desarrollo humano positivo y experiencias enriquecedoras, la adolescencia es, con frecuencia, una etapa proclive a generar comportamientos que afectan negativamente a la salud (De Bourdeaudhuij y Van Oost, 1998), añadiendo al aburrimiento, a la falta de ejercicio físico o al 
descanso inadecuado, prácticas que derivan en violencia, delincuencia o consumo abusivo de sustancias nocivas como el alcohol, el tabaco u otras drogas (Sharp et al., 2006).

Aludimos, por tanto, a un variado conjunto de circunstancias que ponen énfasis en la necesidad de promover una educación del ocio que optimice el crecimiento personal y social de los individuos. Al respecto, la World Leisure and Recreation Association, promotora de la Carta Internacional para la Educación del Ocio (1993), considera que el objetivo general de dicha educación debe consistir, entre otros aspectos, en ayudar a que los jóvenes conquisten una deseable calidad de vida a través de su tiempo libre, mediante el fomento y el desarrollo de valores, actitudes, conocimientos y habilidades que incidan en los modos de socializarse. Una Educación del Ocio que invoca valores significativos para el desarrollo humano, la innovación y transformación social, en una sociedad red cada vez más necesitada de estímulos pedagógicos, culturales y sociales (Caride, 2012).

\section{EL OCIO FÍSICO-DEPORTIVO COMO ÁMBITO PRIVILE- GIADO PARA EL CRECIMIENTO PERSONAL}

Son muchas las investigaciones que han destacado el valor educativo del ocio físico-deportivo para el desarrollo personal y social de jóvenes y adolescentes (Blasco et al., 1996; Duda y White, 1992; Gutiérrez Sanmartín, 2004).

Más en concreto, el Consejo Superior de Deportes (2010a, 2010b) verifica que el deporte constituye un valioso instrumento de integración social, que facilita el acercamiento entre personas de distintas nacionalidades y culturas, así como la promoción de hábitos solidarios y actitudes inclusivas, beneficiando, por ende, la calidad de las relaciones humanas.

Se confirma, además, que la práctica de actividad física aumenta las destrezas sociales, mejora la comunicación y los procesos de liderazgo (Reitman, O'Callaghan y Mitchell, 2005), agrandando las interacciones grupales y la cooperación entre iguales, al tiempo que reduce los desajustes, la vulnerabilidad y el aislamiento social. En este sentido, diferentes investigaciones (Griffin, 1985; Moreno y Hellín, 2002; Velázquez y Hernández, 2003) ratifican que los jóvenes destacan el valor de la amistad y las relaciones sociales en su actividad física, siendo variables influyentes en su promoción y mantenimiento.
Se constata, asimismo, que el ocio físico-deportivo impulsa valores orientados a optimizar el desarrollo personal, siendo una práctica que además de favorecer la autoestima, el autoconcepto y la autoconfianza (Ponce de León et al., 2009; Ruiz de Azúa, Rodríguez y Goñi, 2005), contribuye a que los individuos se sientan más seguros de sí mismos y de su aspecto, que se relaciona con una menor exposición a comportamientos de riesgo y a hábitos de vida insalubres (Nelson y Gordon-Larsen, 2006). El estado emocional también guarda una estrecha relación con la actividad física, tanto en su cantidad como en su calidad. Autores como Sacker y Cable (2006) asocian el bienestar psicológico del adulto a las prácticas de ocio físico-deportivo que realizaron en su adolescencia; a su vez, Ussher, Owen et al., (2007) ponen de manifiesto una fuerte relación entre un bajo estado de bienestar emocional en el adolescente y un nivel de actividad física reducido.

\section{LA FAMILIA COMO INSTITUCIÓN UNIVERSAL $Y$ PLURAL EN LA SOCIEDAD RED}

La sociedad del siglo XXI se identifica con la sociedad de la información y la comunicación, con espacios y tiempos cada vez más condicionados por los constantes y vertiginosos cambios que en ella acontecen, entre lo real y lo virtual. Un conjunto de circunstancias en las que inscribe sus realidades la institución familiar, dando como resultado una compleja diversidad de estructuras y tipos de familia que dificulta su definición, al menos si se pretende que sea válida para todas sus modalidades, tanto en sus elementos socioculturales como biológicos. Como diría Iglesias de Ussel (1998, p.293), aun siendo "la más universal de las instituciones sociales... sus formas históricas han sido demasiado diversas para poder subsumirlas en un único concepto". La familia, en realidad, son familias. Que esto suceda no solo comporta que estén cambiando los modos de entenderlas, sino y, sobre todo, cómo se han ido incorporando a sus concepciones las importantes diferencias de carácter transcultural que las informan (Álvarez y Rodríguez, 2008), en sus funciones, organización y estructura relacional. Las nuevas formas de articulación de las familias no implica la desaparición de la familia, sino una mayor apertura del sistema familiar a la heterogeneidad, tanto en su composición y tamaño (familia troncal tradicional o convencional, monoparental, polinuclear, parejas de hecho, etc.) o en las relaciones que mantiene con otras estructuras socio-institucionales, desde el Estado hasta la Iglesia, pasando por la escuela, los servicios sociales, etc. 
Con carácter general, puede decirse que la familia -las familias- son sistemas formalizados, abiertos, propositivos y autorregulados, que llevan inherentes unas estructuras jerárquicas entre sus miembros, reguladas por normas que delimitan sus relaciones internas y externas, que tienen como finalidad salvaguardar la pertenencia de sus miembros, a los que unen vínculos de afecto e intimidad (Álvarez y Rodríguez, 2008; García-Roca, 1999; Romero, 2002).

Al margen de otras consideraciones, su caracterización como una célula básica de la sociedad -en palabras de Augusto Comte- sigue reflejándose en el hecho de ser la institución más valorada por todos los ciudadanos, cualquiera que sea su edad, género o clase social: un ámbito privilegiado para la convivencia y la transmisión de saberes fundamentales relacionados con la socialización en la vida cotidiana, que propician o inhiben la transferencia de hábitos, habilidades y pautas de convivencia (Elzo, 2004).

Apoyándonos en Satir (2007), uno de los principales referentes en las investigaciones en el campo de la terapia familiar, diremos que aquellas familias que promuevan y respeten las diferencias individuales, que acepten los errores, en las que exista comunicación entre todos sus miembros y con el entorno, y que cuenten con un sistema de reglas de convivencia flexible, contribuirán al desarrollo de jóvenes más plenos, lo que indica que los tiempos y los espacios familiares son un terreno propicio para la construcción del ocio como una experiencia de desarrollo humano estimable y valiosa (Cuenca, 2013).

Esta circunstancia recuerda, como ya hemos anticipado, que la institución familiar goza de un gran aprecio social; tanto que a pesar de los cambios que se viene experimentando en las últimas décadas, sigue siendo valorada como uno de los pilares fundamentales de la vida personal y social: un núcleo cardinal con alta capacidad pedagógica, en la que se gesta el proceso de socialización del individuo y su educación en valores, que ayuda a la orientación vital y proporciona estabilidad (Álvarez y Rodríguez, 2008). Complementariamente, abundan los estudios han constatado la importancia del papel de los padres como germen de apoyo en el ajuste emocional y conductual de los hijos (Demaray y Malecki, 2002; Jackson y Warren, 2000; Juang y Silbereisen, 1999).

No obstante, los diferentes tipos y modelos de familia que coexisten en la sociedad contemporánea obligan a replantear algunas de las convenciones en las que nos hemos ido instalando en el pasado, entre otras: que la familia nuclear ya no es imprescindible para el establecimiento de unas relaciones familiares positivas y un desarrollo psicológico saludable en el joven (Pérez Milena et al., 2007); o que la estructura familiar ha dejado de ser un factor decisivo para una adecuada percepción, por parte de los adolescentes, de las funciones que cabe atribuirle a las familias (Pérez Milena et al., 2009). Dos cuestiones que al poner en relación a las familias con el ocio juvenil, acentúan el interés de una línea de investigación con un creciente protagonismo en las Ciencias Sociales y de la Educación. En síntesis: todo indica que el rol materno-parental ocupa un lugar relevante en la construcción adecuada del ocio de los hijos, en general, y del ocio físico-deportivo, en particular, desde la infancia hasta la juventud.

\section{VARIABLES FAMILIARES SUSCEPTIBLES DE INFLUIR EN EL OCIO FÍSICO-DEPORTIVO JUVENIL}

La literatura científica evidencia que las madres y los padres juegan un importante papel en la motivación para la actividad física de sus hijos adolescentes en el espacio y el tiempo de ocio (McDavid, Cox y Amorose, 2011; Piéron y Ruiz Juan, 2013; Sanz, Ponce de León y Valdemoros, 2012; Tuero, Zapico y González, 2012; Van der Horst et al., 2010).

Al respecto, y entre las líneas empíricas que han focalizado su atención en las variables familiares que ejercen algún tipo de influjo en la construcción del ocio juvenil, se señalan cinco aspectos o dimensiones en los que las realidades familiares tienen un especial significado, principalmente: el estilo educativo familiar, el nivel educativo y/o económico de los padres, los estilos de vida parentales, la estructura familiar y el funcionamiento familiar. Nos detendremos, brevemente, en cada una de ellas:

a) En lo que atañe al estilo educativo familiar son muchas las contribuciones, nacionales e internacionales, que afirman que la participación de los jóvenes en unas $u$ otras actividades de ocio se relacionan con los estilos educativos familiares (Borawski et al., 2003; Bumpus, Crouter, McHale, 2001; Kristjansson et al., 2010; Pate et al., 2011; Smetana y Daddis, 2002; Tomás, Tort y Costa, 2007), determinando que cuando los padres-madres son permisivos con sus hijos es más fácil que los jóvenes adopten estilos de ocio poco saludables o adictivos, como el consumo de alcohol y otras drogas, o el uso abusivo de televisión, Internet, consolas o videojuegos. Al respecto, cabe destacar que mientras unos autores afirman que un control por parte 
de los padres sobre lo que hacen sus hijos se asocia con un ocio más saludable y menos sedentario de sus descendientes (Carlson et al., 2010; Lin, Lin y Wu, 2009), otros detectan que la vigilancia de los padres ejerce un efecto negativo en el ocio de sus hijos (Pieron y Ruiz-Juan, 2013; Sharp et al., 2006). Las discrepancias en los resultados obtenidos evocan la necesidad de continuar indagando en estas variables familiares.

b) En referencia al nivel educativo y/o económico de los padres, son abundantes los estudios que analizan la relación existente entre estas variables y las actividades de ocio de los hijos. Tampoco aquí los investigadores se ponen de acuerdo, puesto que se encuentran trabajos que señalan una asociación significativa entre el ocio de los jóvenes de ambos sexos y el nivel educativo y/o económico de los dos progenitores (Gorely et al., 2009; Piko y Keresztes, 2008; Van der Horst et al., 2007), frente a otros que detectan cómo tan solo el nivel educativo o económico de uno de los dos, padre o madre, se relaciona con las actividades de ocio de sus hijos e hijas o tan solo de alguno de ellos (Rangul et al., 2011; Toftegaard-Stockel et al., 2011; Wight et al., 2009). Incluso, algunas investigaciones recientes apuntan que no existe ninguna concordancia significativa entre el nivel cultural y/o económico de los padres y el tipo de ocio de los jóvenes (Sanz, Ponce de León y Valdemoros, 2012). La disparidad de resultados obliga a dar continuidad a la investigación de estos factores parentales, susceptibles de influir en el ocio físico-deportivo de los hijos.

c) Continuando con la variable estilo de vida de los padres, los estudiosos del ocio físico-deportivo tampoco coinciden en sus hallazgos (análisis, resultados, etc.) observándose dos tendencias principales: de un lado, los que refuerzan la teoría de que los padres se constituyen en modelo por excelencia para el aprendizaje de valores, actitudes y comportamientos de sus hijos, al concluir que la práctica físico-deportiva de ambos progenitores se relaciona con la de los hijos, tanto chicas como chicos (Cantallops et al., 2012; Fuemmeler, Anderson y Mâsse, 2011; Richards et al., 2009; Pieron y Ruiz-Juan, 2013; Ruiz-Juan, Piéron y Baena-Extremera, 2012; ToftegaardStockel et al., 2011; Van der Horst et al., 2010); de otro, los que indican que la actividad física de padres y madres se relaciona con la de sus hijas pero no con la de sus hijos (Dumith et al., 2010); a los que se añaden investigaciones que ponen de relieve que solo la ejercitación de la madre, no la del padre, se asocia con la actividad física de los hijos de ambos géneros (Kahn et al., 2008) o, incluso, favorece que su hija, no su hijo, deje de ser inactiva (Rangul et al., 2011). Una vez más, ante la divergencia de resultados, se hace necesario prolongar la investigación en torno a este tipo de factores, susceptibles de condicionar la actividad física del adolescente en los espacios y tiempos de ocio.

d) Cuando se toma en consideración la estructura familiar todo indica, en la sociedad del siglo $X X I$, que estamos obligados a reinventar la institución familiar, reconociendo la coexistencia de múltiples tipos de familia (Bolívar, 2006), siendo numerosos los estudios que analizan la relación que se da entre la estructura familiar y el ocio de los jóvenes. Algunos autores han llegado a constatar que quienes pertenecen a familias monoparentales manifiestan un mayor comportamiento sedentario que aquellos que viven en familias biparentales (Gorely et al., 2009; Thérond, Duyme y Capron, 2002). Por el contrario, Domínguez y Arroyo (2001) comprueban que este hecho no ha de implicar necesariamente problema alguno, constituyéndose en factores de riesgo las desventajas económicas, la pobreza, una inadecuada comunicación o los problemas afectivos. En la misma línea, Pérez Milena et al. (2007) verifican que la estructura familiar no condiciona el ocio de los hijos, dado que no influye ni en la sensación de apoyo social que estos experimentan, en el consumo de sustancias, en la presencia de indicios de malestar emocional, por lo que se estima que la atención familiar en esta época ha de focalizarse en el fomento de una dinámica familiar positiva, independientemente de la estructura familiar (Pérez Milena et al., 2009).

e) Por último, se aborda el funcionamiento familiar como una variable susceptible de influir en el ocio de los hijos e hijas, siendo especialmente significativa su incidencia en las prácticas físicodeportivas. Cabe destacar que en el estudio que estamos realizando -"De los tiempos educativos a los tiempos sociales: la cotidianidad familiar en la construcción del ocio físico-deportivo juvenil"se utilizará como referente el Modelo Circumplejo de Sistemas Familiares y Maritales (Olson, 2000; Olson, Sprenkle y Russell, 1979), al ser uno de los que mayor repercusión ha experimentado en los últimos años en la esfera académica; lo hacemos en la medida en que integra diversos conceptos a los que se recurre en la terapia familiar, enfatizando la necesidad de valorar el funcionamiento familiar atendiendo a tres variables o constructos 
fundamentales: la cohesión, la flexibilidad y la comunicación. A ellos se agregan otros conceptos (Martínez-Pampliega, Iraurgi y Sanz, 2011) que ayudan a su explicación y comprensión.

La cohesión será entendida como la reciprocidad emocional que se da o puede darse entre los miembros de la familia, queda asociada a los lazos familiares, a la implicación familiar, al respeto mutuo entre padres e hijos, o al establecimiento de "fronteras internas" en las relaciones intergeneracionales.

La flexibilidad se interpreta como la capacidad para hacer frente, de modo adecuado, a los cambios y/o adaptaciones que se requieran en una determinada coyuntura, aprendiendo de las diferentes experiencias y situaciones a las que puedan dar lugar, a menudo con algún tipo de consecuencia práctica en los procesos de liderazgo, la negociación, la disciplina, los roles o las reglas que adopten las personas que participen en ellas.

Por último, la comunicación se concibe como el acto de dar a conocer a los distintos miembros de la familia las informaciones que pueden y/o deben compartir: ideas, pensamientos, sentimientos, decisiones, etc. que les afectan, facilitando o inhibiendo la cohesión y la flexibilidad que se precisa para satisfacer las necesidades y demandas que surjan. Entre los conceptos ligados a la comunicación cabe destacar la empatía y la escucha activa por parte del receptor, las habilidades del emisor, la libertad y la claridad de expresión, la continuidad, la lealtad y la consideración.

De todo ello se infiere que un sistema familiar tendrá un funcionamiento poco saludable cuando exista falta de comunicación, sea muy poco o demasiado flexible y/o cuando la dependencia al grupo sea excesiva, de lo que resulta un sistema de desequilibrios, incapaz de dar respuesta a las demandas que emergen en una sociedad tan cambiante como la que habitamos (Smith, Freeman y Zabriskie, 2009). Además, se constata que tanto una buena comunicación entre padres e hijos (Sharp et al., 2006; Smith, Freeman y Zabriskie, 2009) como una adecuada cohesión y flexibilidad familiar (Hornberger, Zabriskie y Freeman, 2010; Smith et al., 2009) favorecen un ocio juvenil y familiar positivo en el que se disfrutan experiencias interesantes, atractivas y enriquecedoras, lo que se relaciona significativamente con una mayor satisfacción en la vida familiar (Agate et al., 2009). Asimismo, investigadores españoles (Ruiz-Juan, Piéron y Baena-Extremera, 2012) verifican que cuando una familia carece de un patrón de estilo de vida común y coherente, puede esperarse escasa probabilidad de que los hijos adquieran hábitos de vida activos. De ahí que podamos afirmar que la convivencia interna familiar es la única variable de las analizadas que ofrece sintonía en sus hallazgos, ratificando que la cohesión, la flexibilidad y la comunicación son tres factores decisivos en la construcción y consolidación de un ocio físicodeportivo integrador, ya desde las primeras etapas evolutivas del ser humano.

\section{CONCLUSIONES}

La reflexión científica ofrece conclusiones discrepantes sobre la vinculación entre el estilo educativo familiar, el nivel educativo y/o económico de los padres, el comportamiento modelo de los padres o la estructura familiar, y el ocio de los hijos e hijas adolescentes. Hay estudios que relacionan el control parental con estilos de vida saludable y otros lo desligan; otras investigaciones vinculan el nivel educativo y económico de los progenitores con la actividad de ocio, mientras que otras lo disocian. De ahí que podamos afirmar que no se existe unanimidad acerca de la relación que existe entre los estilos de vida de los progenitores y el de sus descendientes; finalmente, tampoco hay consenso acerca de si el modelo de familia es una variable condicionante.

Frente a ello, coincidiendo con nuestra hipótesis de partida, la literatura existente centra su atención en el funcionamiento interno (o, si se prefiere, en la convivencia familiar), como una variable contextual, susceptible de mediar y condicionar la adquisición y/o la consolidación de los estilos de vida de los jóvenes y adolescentes en las sociedades avanzadas (Agate et al., 2009; Hornberger, Zabriskie y Freeman, 2010; Kitzman-Ulrich et al., 2010; Sharp et al., 2006; Smith, Freeman y Zabriskie, 2009; Van Ryzin y Nowicka, 2013).

Más en concreto: la cohesión, la flexibilidad y la comunicación que se vivencie en las familias, en sintonía con las iniciativas y las estrategias para el fomento de los tiempos compartidos, generarán un contexto idóneo para la construcción de un ocio físico-deportivo como experiencia de desarrollo humano que ayuda al crecimiento personal. Estos factores, también actúan como nexo de unión entre las otras variables objeto de estudio.

En su conjunto, son conclusiones que avalan la necesidad de realizar un estudio de ámbito nacional que detecte los factores familiares que influyen en la construcción del ocio físico-deportivo juvenil, en general, y de aquellos referentes a la convivencia fa- 
miliar, en particular, utilizando como instrumento la versión española de 20 ítems de la Escala de Evaluación de la Cohesión y Adaptabilidad Familiar (FACES20Esp, de Martínez-Pampliega, Iraurgi y Sanz, 2011), dado que se fundamenta en el Modelo Circumplejo de Sistemas Familiares y Maritales (Olson, 2000; Olson, Sprenkle y Russell, 1979), el paradigma familiar con mayor difusión en los últimos años.

Tenemos la convicción de que los resultados a los que se remitan los estudios empíricos que se realicen, aportarán argumentos suficientes para que se le otorgue un mayor protagonismo a estas variables en las políticas educativas, culturales y sociales; sobre todo si se pretende actuar con criterios que doten de una mayor viabilidad al diseño, implementación y evaluación de programas de acción-intervencióneducación familiar que incidan en una doble línea de trabajo: por un lado, neutralizando los factores o circunstancias que dificultan el desarrollo integral de los adolescentes y jóvenes en sus prácticas de ocio; de otro, incrementando los que contribuyan a mejorar su bienestar-individual y social- y calidad de vida. En definitiva, la meta está en lograr que sean autónomos y responsables en y ante su propio ocio, allí donde se concrete; que es lo mismo que decir de su propia vida, presente y futura. También, y por extensión, en lo que ya Norbert Elias y Eric Dunning (1986) entendieron que el deporte y el ocio podrían hacer por mejorarnos como civilización.

\section{AGRADECIMIENTOS}

El texto que presentamos se vincula al Proyecto de Investigación "De los tiempos educativos a los tiempos sociales: la construcción cotidiana de la condición juvenil en una sociedad de redes. Problemáticas específicas y alternativas pedagógico-sociales" (proyecto coordinado EDU2012-39080-C07-00) y al subproyecto "De los tiempos educativos a los tiempos sociales: la cotidianidad familiar en la construcción del ocio físico-deportivo juvenil" (EDU201239080-C07-05), cofinanciado en el marco del Plan Nacional I+D+i con cargo a una ayuda del Ministerio de Economía y Competitividad y del Fondo Europeo de Desarrollo Regional (FEDER, 2007-2013).

Agradecemos al Instituto de Estudios Riojanos el soporte financiero concedido al estudio dentro de las ayudas para estudios científicos de temática riojana (Ref.: Resolución no 110 de 27 de diciembre de 2013 del Gerente del IER).

\section{BIBLIOGRAFÍA}

Agate, J.R., Zabriskie, R.B., Agate, S.T. y Poff, R. (2009).Family Leisure Satisfaction and Satisfaction with Family Life. Journal of Leisure Research, 41, 2, pp. 205-223.

Álvarez Rodríguez, J. y Rodríguez Sabiote, C. (2008). El valor de la institución familiar en los jóvenes universitarios de la Universidad de Granada. Bordón, 60, 1, pp. 7-21

Aristegui, I. y Silvestre, M. (2012). El ocio como valor en la sociedad actual. Arbor, 188, pp. 283-291. http://dx.doi. org/10.3989/arbor.2012.754n2001

Blasco, T., Capdevilla, L., Cruz, J., Pintanel, M. y Valiente L. (1996). Evolución de los patrones de actividad física en estudiantes universitarios. Revista de Psicología del Deporte, 9-10, pp. 51-63.

Bolívar, A. (2006). Familia y escuela: dos mundos llamados a trabajar en común. Revista de Educación, 339, pp. 119-146.

Borawski, E.A., Levers-Landis, C.E., Lovergreen, L.D. y Trapl, E.S. (2003). Paren- ting monitoring, negotiated unsupervised time, and parental trust: the role of perceived parenting practices in adolescent health risk behaviors. Journal of Adolescent Health, 33, pp. 6070. http://dx.doi.org/10.1016/S1054139x(03)00100-9

Bumpus, M.F., Crouter, A.C. y McHale, S.M. (2001). Parental autonomy granting during adolescence: exploring gender differences in context. Developmental Psychology, 37, pp. 163173. http://dx.doi.org/10.1037/0012 1649.37.2.163

Caballo, B., Gradaílle, R. y Merelas, T. (2012). Servicios socioeducativos y corresponsabilidad en la conciliación de los tiempos familiares. Situación de la infancia en la Galicia urbana. Pedagogía Social. Revista Interuniversitaria, 20, pp. 179-202. http://dx.doi. org/10.7179/PSRI_2012.20.5

Cantallops, J., Ponseti, F. J, Vidal, J., Borràs, P. A., y Palou, P. (2012). Adolescencia, sedentarismo y sobrepeso: análisis en función de variables sociopersonales de los padres y del tipo de deporte practicado por los hijos. Retos, 21, pp. 5-8.

Caride, J.A. (1998). Educación del ocio y del tiempo libre. En: Beas, M. y García, J. (coords.), Atención a los espacios y tiempos escolares. Granada: Grupo Editorial Universitario, pp. 17-31.

Caride, J.A. (2012). Lo que el tiempo educa: el ocio como construcción pedagógica y social. Arbor, 188, pp. 301-313. http://dx.doi.org/10.3989/ arbor.2012.754n2001

Carlson, S.A., Fulton, J.E., Lee, S.M., Foley, J. T.; Heitzler, C. y Huhman, M. (2010).Influence of Limit-Setting and Participation in Physical Activity on Youth Screen Time. Pediatrics, 126, 1, pp. 89-96. http://dx.doi.org/10.1542/ peds.2009-3374

Consejo Superior de Deportes (2010a). Plan integral para la actividad física y el deporte [en línea]. [Fecha de consulta: 2 de septiembre de 2013]. Disponible en http://goo.gl/exySuo 
Consejo Superior de Deportes (2010b). Encuesta sobre los hábitos deportivos en España 2010 [en línea]. [Fecha de consulta: 2 de septiembre de 2013]. Disponible en http://goo.gl/TcS2QY

Cuenca, M. (1997). La intervención educativa en ocio y tiempo libre. En AAVV: Nuevos espacios de la educación social. Bilbao: Universidad de Deusto, pp. 253-286.

Cuenca, M. (2002). La educación del ocio. Ámbitos de acción futura. Revista de Educación, número extraordinario, pp. 149-167.

Cuenca, M. (2009). Ocio humanista. Dimensiones y manifestaciones actuales del ocio. Bilbao: Universidad de Deusto.

Cuenca, M. (2013). Ocio valioso en tiempos de crisis. En Torío, S., García-Pérez, O., Peña, J.V. y Fernández, C. (coords.). La crisis social y el estado del bienestar: las respuestas de la pedagogía social. Oviedo: Universidad de Oviedo, pp. 5-20.

Cuenca, M. y Goytia, A. (2012). Ocio experiencial: antecedentes y características. Arbor, 188, pp. 265-281. http://dx.doi. org/10.3989/arbor.2012.754n2001

De Bourdeaudhuij, I. y Van Oost, P. (1998). Family characteristics and positive health behaviors in adolescents and families. Psychology and Health, 13, pp. 785-803. http://dx.doi. org/10.1080/08870449808407432

Demaray, M. K. y Malecki, C. K. (2002). The relationship between perceived social support and maladjustment for student at risk. Psychology in the Schools, 39, 3, pp. 305-316. http://dx.doi. org/10.1002/pits.10018

Domínguez, M. y Arroyo, A. (2001). La socialización de los hijos en las familias monoparentales. Revista de Educación, 325, pp. 99-112.

Duda, J. L. y White, S.A. (1992). Goal orientations and beliefs about the causes of sport success among elite skiers. The Sport Psychologist, 6, pp. 334-343.

Dumith, S.C., Domingues, M.R., Gigante, D.P., Hallal, P.C., Menezes, A.M.B. y Kohl, H.W. (2010). Prevalence and correlates of physical activity among adolescents from Southern Brazil. Revista de Saúde Pública, 44, 3, pp. 457-467. http://dx.doi.org/10.1590/S003489102010000300009

Elias, N. y Dunning, E. (1986). Quest for excitement. Sport and Leisure in the Ci- vilizing Process. Oxford-New York: Basil Blackwell Publisher.

Elzo, J. (2004). Tipología y socialización de las familias españolas. Arbor, 702, pp. 205-229. http://dx.doi.org/10.3989/ arbor.2004.i702.566

Fraguela, R., Lorenzo, J.J. y Varela, L. (2011). Escuela, familias y ocio en la conciliación de los tiempos cotidianos de la infancia. Revista de Investigación Educativa, 29, 2, pp. 429-446.

Fuemmeler, B.F., Anderson, C.B. y Mâsse, L.C. (2011). Parent-child relationship of directly measured physical activity. International Journal of Behavioural $\mathrm{Nu}$ trition and Physical Activity, 8, 17, pp. 1-9. http://dx.doi.org/10.1186/14795868-8-17

García-Roca, J. (1999). Composición y funciones básicas de la familia. En Gimeno, A. La familia: el desafío de la diversidad. Barcelona: Ariel, pp. 33-62.

Gorely, T., Atkin, A.J., Biddle, S.J.H. y Marshall, S.J. (2009). Family circumstance, sedentary behaviour and physical activity in adolescents living in England: Project STIL. International Journal of Behavioral Nutrition and Physical Activity, 6, 33. http://dx.doi. org/10.1186/1479-5868-6-33

Griffin, P. (1985). Boys' participation styles in a middle school physical education team sports unit. Journal of Teaching in Physical Education, 4, pp. 100-110.

Gutiérrez Sanmartín, M. (2004). El valor del deporte en la educación integral del ser humano. Revista de Educación, 335, pp. 105-126.

Hornberger, L.B., Zabriskie, R.B. y Freeman, P. (2010). Contributions of Family Leisure to Family Functioning Among Single-Parent Families. Leisure Sciences, 32, 2, pp. 143-161. http://dx.doi. org/10.1080/01490400903547153

Iglesias de Ussel, J. (1998). Familia. En Giner, S., Lamo de Espinosa, E. y Torres, C. (eds.). Diccionario de Sociología. Madrid: Alianza Editorial, pp. 293-295.

Jackson, Y. y Warren, J. S. (2000). Appraisal, social support, and life events: predicting outcomes behavior in schoolage children. Child Development, 71, 5, pp. 1441-1457. http://dx.doi. org/10.1111/1467-8624.00238

Juang, L. P. y Silbereisen, R. K. (1999). Supportive parenting and adolescent adjustment across time informer East and West German. Journal of Adolescen- ce, 22, 6, pp. 719-736. http://dx.doi. org/10.1006/jado.1999.0267

Kahn, J.A., Huang, B., Gillman, M.W., Field, A.E., Austin, S.B., Colditz, G.A. y Frazier, A.L. (2008). Patterns and determinants of physical ativity in U.S. adolescents. Journal of Adolescent Health, 42, pp. 369-377. http://dx.doi.org/10.1016/j. jadohealth.2007.11.143

Kitzman-Ulrich, H., Wilson, D.K., St. George, S.M., Lawman, H., Segal, M., Fairchild, A. (2010). The Integration of a Family Systems Approach for Understanding Youth Obesity, Physical Activity, and Dietary Programs. Clinical Child and Family Psychology Review, 13, 6, pp. 231-256. http://dx.doi. org/10.1007/s10567-010-0073-0

Kristjansson, A.L., James, J.E., Allegrante, J.P., Sigfusdottir, I.D. y Helgason, A.R. (2010). Adolescent substance use, parental monitoring, and leisure-time activities: 12-year outcomes of primary prevention in Iceland. Preventive Medicine, 51, pp. 168-171. http://dx.doi. org/10.1016/j.ypmed.2010.05.001

Lin, C.H., Lin, S.L. y Wu, C.P. (2009). The effects of parental monitoring and leisure boredom on adolescents' Internet addiction. Adolescence, 44, 176, pp. 993-1004.

Martínez-Pampliega, A.; Iraurgi, I. y Sanz, M. (2011). Validez estructural del FACES-20Esp: Versión española de 20 ítems de la Escala de Evaluación de la Cohesión y Adaptabilidad Familiar. Revista Iberoamericana de Diagnóstico y Evaluación Psicológica, 29, 1, pp. 147-165.

McDavid, L., Cox, A. E. y Amorose, A.J. (2011). The relative roles of physical education teachers and parents in adolescents' leisure-time physical activity motivation and behavior. Psychology of Sport and Exercise, 13, 2, pp. 99-107. http://dx.doi.org/10.1016/j.psychsport.2011.10.003

Moreno, J. A. y Hellín, G. (2002). ¿Es importante la Educación Física? Su valoración según la edad del alumno y el tipo de centro. Revista Internacional de Medicina y Ciencias de la Actividad Física y del Deporte, 8 [en línea]. [Fecha de consulta: 2 de agosto de 2013]. Disponible en http://cdeporte.rediris. es/revista/

Nelson, M. y Gordon-Larsen, P. (2006). Physical activity and sedentary behaviour patterns are associated with 
selected adolescent health risk behaviours. Pediatrics, 117, 4, pp. 1281 1290. http://dx.doi.org/10.1542/ peds.2005-1692

Olson, D.H. (2000). Circumplex model of marital and family systems. Journal of Family Therapy, 22, 2, pp. 144167. http://dx.doi.org/10.1111/14676427.00144

Olson, D., Sprenkle, D. H., y Russell, C. R. (1979). Circumplex Model of marital and family systems. I. Cohesion and adaptability dimensions, family types, and clinical applications. Family Process, 18, pp. 3-28. http://dx.doi.org/10.1111/j.15455300.1979.00003.x

Pate, R.R., Mitchell, J.A., Byun, W. y Dowda, M. (2011). Sedentary behaviour in youth. British Journal of Sport Medicine, 45, 11, pp. 906-913. http://dx.doi. org/10.1136/bjsports-2011-090192

Pérez Milena, A., Martínez Fernández, M. L., Mesa, I., Pérez Milena, R., Leal, F.J. y Jiménez Pulido, I. (2009). Cambios en la estructura y en la función familiar del adolescente en la última década (1997-2007). Atención primaria, 41, 9, pp. 479-486. http://dx.doi. org/10.1016/j.aprim.2009.03.015

Pérez Milena, A., Pérez Milena, R., Martínez Fernández, M. L., Leal, F. J., Mesa, I. y Jiménez Pulido, I. (2007). Estructura y función de la familia durante la adolescencia: relación con el apoyo social, el consumo de tóxicos y el malestar psíquico. Atención primaria, 39, pp. 61-65. http://dx.doi.org/10.1157/13098670

Piéron, M.; Ruiz Juan, F. (2013). Influencia del ámbito familiar e iguales en los hábitos físico-deportivos de los jóvenes. Revista Internacional de Medicina y Ciencias de la Actividad Física y del Deporte, 51, pp. 525-549.

Piko, B. y Keresztes, N. (2008). Sociodemographic and socioeconomic variations in leisure time physical activity in a sample of Hungarian youth. International Journal of Public Health, 53, pp. 306-310. http://dx.doi.org/10.1007/ s00038-008-7119-9

Ponce de León, A., Sanz, E., Valdemoros, M.A., y Ramos, R. (2009). Los valores personales en el ocio físico-deportivo. Un estudio con jóvenes, padres y profesores. Bordón, 61, 1, pp. 29-41.

Quintana, J.M. (1991). El tiempo libre como ámbito humano y cultural. En Quintana, J.M. Iniciativas sociales en educación informal. Madrid: Rialp, pp. 402-415.

Rangul, V., Holmen, T.L., Bauman, A., Bratberg, G.H., Kurtze, N. y Midthjell, K. (2011). Factors predicting changes in physical activity through adolescence: the young-HUNT study, Norway. Journal of Adolescent Health, 48, pp. 616-624. http://dx.doi.org/10.1016/j. jadohealth.2010.09.013

Reitman, D., O'Callaghan, P. M. y Mitchel, P. (2005). Parent as coach: enhancing sports participation and social behaviour for ADHD-diagnosed children. Child and Family Behavior Therapy, 27, 2, pp. 57-68. http://dx.doi. org/10.1300/J019v27n02_06

Richards, R., Poulton, R., Reeder, A.I., y Williams, S., (2009). Childhood and Contemporaneous Correlates of Adolescent Leisure Time Physical Inactivity: A Longitudinal Study. Journal of Adolescent Health, 44, pp. 260-267. http://dx.doi.org/10.1016/j.jadohealth.2008.08.005

Roberts, K. (2012). El ocio de los jóvenes en la sociedad contemporánea. Arbor, 188, pp. 327-337. http://dx.doi. org/10.3989/arbor.2012.754n2001

Romero, C. (2002). La familia desde una perspectiva sistémico-dinámica. En Gervilla, E. (coord.). Educación familiar. Nuevas relaciones humanas y humanizadoras. Madrid: Narcea, pp. 155-165.

Ruiz de Azúa, S., Rodríguez, A. y Goñi, A. (2005). Variables socioculturales en la construcción del autoconcepto físico. Cultura y Educación, 17, 3, pp. 225-238. http://dx.doi. org/10.1174/113564005774462582

Ruiz-Juan, F., Piéron, M., y Baena-Extremera, A. (2012). Socialización de la actividad físico-deportiva en adultos: relación con familia, pareja y amigos. Revista Iberoamericana de Diagnóstico y Evaluación Psicológica, 34, 1, pp. 34-59.

Sacker, A. y Cable, N. (2006). Do adolescent leisure-time physical activities foster health and web-being in adulthood? Evidence from two British birth cohorts. European Journal of Public Health, 16, 3, pp. 331-335. http:// dx.doi.org/10.1093/eurpub/cki189

Sanz, E., Ponce de León, A. y Valdemoros M.A. (2012). Parental predictors of physical inactivity in Spanish adolescents. Journal of Sports Science and Medicine, 11, 1, pp. 95-101.
Satir, V. (2007). Terapia familiar paso a paso. Mexico: Editorial Pax México.

Schnabel, U. (2011). Ocio: la felicidad de no hacer nada. Barcelona: Plataforma editorial.

Sharp, E.H., Caldwell, L.L., Graham, J.W. y Ridenour, T.A. (2006). Individual motivation and parental influence on adolescents' experiences of interest in free time: a longitudinal examination. Journal of Youth and Adolescence, 35, 3, pp. 359-372. http://dx.doi. org/10.1007/s10964-006-9045-6

Smetana, J.G. y Daddis, C. (2002). Domain-specific antecedents of parental psychological control and monitoring: the role of parenting beliefs and practices. Child Development, 73, pp. 563580. http://dx.doi.org/10.1111/14678624.00424

Smith, K.M., Freeman, P.A. y Zabriskie, R. B. (2009). An Examination of Family Communication within the Core and Balance Model of Family Leisure Functioning. Family Relations, 58, 1, pp. 79-90. http://dx.doi.org/10.1111/ j.1741-3729.2008.00536.x

Thérond, C., Duyme, M. y Capron, C. (2002). Socioeconomical status (SES) and children behaviour disorders. VIII Conference of the European Association for Research on Adolescence. EARA. Oxford.

Tobío, C. (2002). Conciliación o contradicción: cómo hacen las madres trabajadoras. Revista Española de Investigaciones Sociológicas, 97, pp. 155-186.

Toftegaard-Stockel, J., Nielsen, G.A., Ibsen, B. y Andersen, L.B. (2011).Parental, socio and cultural factors associated with adolescents' sports participation in four Danish municipalities. Scandinavian Journal of Medicine and Science in Sports, 21, pp. 606-611. http://dx.doi.org/10.1111/j.16000838.2010.01093.x

Tomás, M.T.C., Tort, B.E. y Costa, J.A.G. (2007). Characteristics that define the "botellón" phenomenon in university students and adolescents. Adicciones, 19, 4, pp. 357-372.

Torres, R. (2007): Metáforas sociales del tiempo en España. Una investigación empírica. En Prieto, C. (coord.), Trabajo, género y tiempo social. Madrid: Editorial de la Universidad Complutense, pp. 173-204. 
Tuero, C., Zapico, B., y González, B. (2012). Deporte en edad escolar y agentes sociales: estudio preliminar sobre la relación entre familia, monitores y deportistas en la provincia de León. Retos, 21, pp. 34-37.

Ussher, M.; Owen, C. G.; Cook, D. y Whincup, P. (2007). The relationship between physical activity, sedentary behavior and psychological wellbeing among adolescents. Social Psychiatry and Psychiatry Epidemiology, 42, pp. 851-856. http://dx.doi.org/10.1007/ s00127-007-0232-x

Van der Horst, K., Oenema, A., Te Velde, S.J. y Brug, J. (2010). Do individual cognitions mediate the association of socio-cultural and physical environmental factors with adolescent sports participation? Public Health Nutrition, 13, 10A, pp. 1746-1754. http://dx.doi. org/10.1017/S1368980010002314

Van Der Horst, K., Paw, M.J.C.A., Twisk, J.W.R. y Van Mechelen, W. (2007). A brief review on correlates of physical activity and sedentariness in youth. $\mathrm{Me}$ dicine and Science in Sports and Exercise, 39, 8, pp. 1241-1250. http://dx.doi. org/10.1249/mss.0b013e318059bf35

Van Ryzin, M.J. y Nowicka, P. (2013). Direct and Indirect Effects of a FamilyBased Intervention in Early Adolescence on Parent - Youth Relationship Quality, Late Adolescent Health, and Early Adult Obesity. Journal of Family Psychology, 27, 1, pp. 106-116. http:// dx.doi.org/10.1037/a0031428
Velázquez, R. y Hernández, J. L. (2003). Deporte y formación de actitudes y valores en los niños, niñas y adolescentes de la comunidad de Madrid. Revista de Educación, 331, pp. 369-398.

Wight, V.R., Price, J., Bianchi, S.M. y Hunt, B.R. (2009). The time use of teenagers. Social Science Research, 38, pp. 792809. http://dx.doi.org/10.1016/j.ssresearch.2009.05.009

World Leisure and Recreation Association (1993). International Charter for Leisure Education. European Leisure and Recreation Association (ELRA), Summer 1993, pp. 13-16. 